\title{
Managing Cutaneous Immune-Mediated Diseases During the COVID-19 Pandemic
}

\author{
Tiago Torres ${ }^{1,2}$ (1) $\cdot$ Luis Puig $^{3}$ ()
}

Published online: 10 April 2020

C) Springer Nature Switzerland AG 2020

\begin{abstract}
Coronavirus disease 2019 (COVID-19) is a clinical syndrome caused by a novel coronavirus called severe acute respiratory syndrome coronavirus 2 (SARS-CoV-2). COVID-19 has spread rapidly worldwide and has been shown to have a wide spectrum of severity. COVID-19 has become a public health emergency of relevant international concern, and it was declared a pandemic by the World Health Organization on 11 March, 2020. SARS-CoV-2 infection in severe cases involves the host response as an important contributor to the disease process and tissue damage, mainly due to dysregulated and excessive innate immune responses. The primary immune response leads to viral clearance in the majority of cases. However, in a subgroup of patients, the secondary immune response may be exaggerated, leading to inflammatory-induced lung injury and other complications including pneumonitis, acute respiratory distress syndrome, respiratory failure, shock, organ failure, and potentially death. Several cutaneous immune-mediated diseases, including psoriasis, atopic dermatitis, and hidradenitis suppurativa, are therapeutically managed with biologic and non-biologic immunosuppressive and immunomodulatory drugs. The outbreak of COVID-19 affects the management of these chronic conditions, not only for those who are already receiving treatment but also for those who are about to start a new treatment to control their disease. In this article, the management of cutaneous immune-mediated diseases during the COVID-19 pandemic is discussed.
\end{abstract}

\section{Key message}

The outbreak of coronavirus disease 2019 (COVID-19) affects the management of several cutaneous immunemediated chronic diseases, including psoriasis, atopic dermatitis, and hidradenitis suppurativa, not only for those who are already receiving treatment but also for those who are about to start a new treatment to control their disease.

The host's dysregulated and excessive innate immune response is an important contributor to the disease process and tissue damage in severe cases of severe acute respiratory syndrome coronavirus 2 infection.

Some selective immunosuppressants or immunomodulatory drugs might be useful in controlling the "cytokine storm" associated with a poor outcome of COVID-19.

Tiago Torres

torres.tiago@outlook.com

1 Department of Dermatology, Centro Hospitalar Universitário Do Porto, Porto, Portugal
Coronavirus disease 2019 (COVID-19) is a clinical syndrome caused by a novel coronavirus called severe acute respiratory syndrome coronavirus 2 (SARS-CoV-2). On 31 December, 2019, a cluster of pneumonia cases of unknown etiology was reported in Wuhan, Hubei Province, China. On 9 January, 2020, the China Center for Disease Control and Prevention reported a novel coronavirus as the causative agent of this outbreak. COVID-19 has spread rapidly worldwide since it was first identified in Wuhan and has been shown to have a wide spectrum of severity [1]. COVID-19 has become a public health emergency of relevant international concern, and it was declared a pandemic by the World Health Organization on 11 March, 2020; by the end of that month, more than 750,000 cases and 36,500 deaths had been reported worldwide [2].

Although COVID-19 appears to have a lower severity and mortality rate than two other previous human coronavirus (CoV) infections (SARS-CoV and MERS-CoV), a

2 Instituto de Ciências Biomédicas Abel Salazar, University of Porto, Porto, Portugal

3 Department of Dermatology, Hospital de La Santa Creu I Sant Pau, Barcelona, Spain 
subgroup of patients (particularly elderly people and those with underlying medical co-morbidities) develop a severe disease characterized by interstitial pneumonia and the rapid development of acute respiratory distress syndrome or septic shock with high levels of acute-phase reactants $[3,4]$.

Highly pathogenic human $\mathrm{CoV}$ infections suppress type I interferon (IFN) production and signaling, the key component of host antiviral defense in the early phase of viral replication, thus exacerbating disease progression [5]. In line with SARS and MERS, SARS-CoV-2 infection in severe cases involves the host response as an important contributor to the disease process and tissue damage, mainly due to dysregulated and excessive innate immune responses. The primary immune response leads to viral clearance in most cases. However, for reasons that are still unclear, in a subgroup of patients, the secondary immune response may be exaggerated, leading to inflammatory-induced lung injury and complications including pneumonitis, acute respiratory distress syndrome, respiratory failure, shock, organ failure, and potentially death [6]. This exaggerated response is considered a "cytokine storm" similar to that of secondary hemophagocytic histiocytosis, which leads to an hyperinflammation state, with unremitting fever, cytopenias, hyperferritinemia, and pulmonary involvement (including acute respiratory distress syndrome) in 50\% of cases [7]. The cytokine profile associated with COVID-19 is characterized by elevated serum levels of multiple cytokines [3], probably produced by highly inflammatory macrophages [8]. Interleukin (IL)-6, IL-10, and tumor necrosis factor (TNF)- $\alpha$ surge during illness and decline upon recovery; overproduction of IL-6, IL-10, soluble IL-2 receptor, and TNF $\alpha$ is inversely correlated with CD4+ and CD8+ T-cell counts, is associated with decreased IFN- $\gamma$ expression in CD4+ cells, and correlates with the disease severity of COVID-19 [8, 9]. In severe cases, marked decreases in memory helper $\mathrm{T}$ cells and regulatory $\mathrm{T}$ cells have been observed [10].

The most commonly reported clinical symptoms of COVID-19 among approximately 14,000 cases reported to the European Surveillance System have been fever (47\%), dry or productive cough $(25 \%)$, sore throat $(16 \%)$, general weakness (6\%), and pain (5\%) [1]. Diagnostic suspicion is generally made on clinical signs and symptoms (fever, fatigue, dry cough, anorexia, dyspnea, rhinorrhea, ageusia, anosmia) and confirmed by polymerase chain reaction tests on nasopharyngeal and oropharyngeal swabs or sputum. There are preliminary reports of skin involvement (erythematous rashes, widespread urticaria, and chickenpox-like vesicles) occurring in $20 \%$ of patients in the course of the disease [11]. Hospitalization, generally due to pneumonitis, occurred in $30 \%$ of cases; severe illness (requiring intensive care unit and/or respiratory support) was reported in $15 \%$ of hospitalized cases, and death in $12 \%$. Both hospitalization and mortality rates increase steeply in patients aged 60 years and above [1].

Pulse oximetry, respiratory frequency, and chest X-ray films determine the clinical management of patients and the need for hospitalization. Multiple therapeutic approaches have been proposed and are being studied in clinical trials, including antiviral agents and hydroxychloroquine [12-14]; widespread uptake of the latter has resulted in supply restrictions for dermatologic patients with indications for antimalarials [15]. Corticosteroids are generally contraindicated in the treatment of COVID-19 [16], but the underlying cytokine storm has provided the basis for therapeutic attempts with selective immunosuppressants such as TNF $\alpha$ inhibitors, Janus kinase inhibitors, anti-IL-1 agents, and anti-IL-6 drugs such as tocilizumab $[17,18]$.

Several cutaneous immune-mediated diseases, including psoriasis, atopic dermatitis, and hidradenitis suppurativa, are therapeutically managed with biologic and non-biologic immunosuppressive and immunomodulatory drugs. The outbreak of COVID-19 affects the management of these chronic conditions, not only for those who are already receiving treatment but also for those who are about to start a new treatment to control their disease.

Most recommendations from dermatology societies [19-21], but also from other specialties that treat immunemediated diseases (rheumatology [22] and gastroenterology [23]) recommend patients to discontinue or postpone immunosuppressive or biologic therapy in the event of COVID-19 diagnosis until the patient recovers from the infection. However, the decision to stop or withhold treatment is left to the joint consideration of the patient and physician, pondering the risk/benefit on a case-by-case basis depending on the risk of SARS-CoV-2 infection.

There is no available evidence that supports discontinuation of biologic treatment of psoriasis for most patients simply because of the risk of infection [24]. According to the results of randomized clinical trials of biological agents in psoriasis, the rates of nasopharyngitis and upper respiratory tract infections are similar to placebo [25].

Some authors have proposed a classification of immunomodulators and immunosuppressants according to "likely risk", suggesting continuation of TNF $\alpha$ inhibitors and antiIL biologics ("likely moderate risk") in the presence of mild viral symptoms [26]. Other authors recommend a limitation or avoidance of ciclosporin, methotrexate, and TNF $\alpha$ inhibitors for dermatologic patients in areas with high infection rates and stopping all immunosuppressive and biological therapy when exposure to confirmed COVID-19 cases occurs [27]. Finally, some authors propose that the use of biologic therapies that modulate $\mathrm{T}$ helper (Th)-1 responses, such as TNF $\alpha$ inhibitors abatacept and ustekinumab, might specifically require more stringent precautions [28]. 
In rheumatology, poor control of rheumatoid arthritis might imply an even greater infectious risk factor than continuation of treatment [29], and the use of systemic corticosteroids might contribute to viral replication in the event of SARS-CoV-2 infection [30]. Immunosuppressive medications in transplanted patients increase the risk or duration of infections caused by viral agents, such as adenovirus, influenza, norovirus, rhinovirus, or respiratory syncytial virus [31]; the same effect does not seem to be observed for infections caused by the coronavirus family. Similarly to SARS-CoV and MERS-CoV [32], immunosuppressed status (due to transplantation, chemotherapy, or other conditions requiring immunosuppressive treatment) has not been found to be a risk factor for an adverse outcome, such as death or admission to an intensive care unit, in patients with COVID19 compared with the general population [33]. Data from a large European center for pediatric liver transplantation located in Lombardy demonstrated that for patients in the follow-up for cirrhosis, transplantation, autoimmune liver disease, or chemotherapy for hepatoblastoma, despite some testing positive for SARS-CoV-2, none developed clinical pulmonary disease [34].

Some selective immunosuppressants or immunomodulatory drugs might be useful in controlling the "cytokine storm" associated with a poor outcome of COVID-19.

Tumor necrosis factor- $\alpha$ levels are moderately increased in patients with SARS, but higher levels have been observed in patients with COVID-19 and correlate with disease severity [35]. Tumor necrosis factor inhibitors might be effective in reducing SARS-CoV2 infection and the associated organ damage, by decreasing TNF- $\alpha$-converting enzymedependent shedding of the ACE2 ectodomain, which is crucial for the penetration of SARS-CoV2 into the cell [36]. A study evaluating adalimumab in COVID-19 infection has recently been registered in the Chinese Clinical Trial Registry (ChiCTR2000030089).

Interleukin-17 plays a crucial role in enhancing antiviral immune responses, but it may also promote and exacerbate viral illnesses, especially through antagonism of regulatory T-cell function, which is of paramount importance in viral infections of the respiratory tract [37]. An elevated Th17 response has also been observed in patients with MERSCoV [38] and SARS-CoV [39]. In SARS-CoV-2, a Th17 response appears to contribute to the "cytokine storm", resulting in tissue damage and pulmonary edema. Extremely high numbers of CCR6p Th17 cells in the peripheral blood of a patient with severe COVID-19 support the role of the Th17 pathway in this disease [40]. Many psoriasis treatments inhibit the IL-17 pathway directly (IL-17A and IL17R inhibitors) and indirectly (IL-23 inhibitors). Low levels of type I IFN seem to be common to COVID-19, SARS, and MERS, and may suppress Th1 and favor Th2 responses [41], thus providing some rationale for the use of dupilumab, an
IL-4/IL-13 inhibitor used in the treatment of atopic dermatitis, to mitigate the "cytokine storm" seen in some patients with COVID-19.

Baricitinib, a Janus kinase 1/2 inhibitor submitted to the US Food and Drug Administration and the European Medicines Agency for the treatment of atopic dermatitis, has been predicted to reduce the ability of the virus to infect lung cells because it targets members of the numb-associated kinase (NAK) family, including adaptor-associated protein kinase 1 (AAK1) and cyclin G-associated kinase (GAK), which are both involved in viral endocytosis [42]. Baricitinib appears to be able to effectively inhibit AAK1 and GAK at the plasma concentration obtained with the submitted dosage for the treatment of atopic dermatitis (2-4 mg daily), as well as reducing IL- 6 and IFN- $\gamma$ levels, which may be responsible for the more severe forms of interstitial pneumonia due to COVID-19 [42, 43]. However, the inhibition of IFN activity and myelopoiesis might be detrimental in these patients [29].

Corticosteroids are not routinely recommended in patients with COVID-19 unless indicated for other reasons (i.e., exacerbation of asthma or chronic obstructive pulmonary disease, septic shock) because they might exacerbate COVID-19-associated lung injury, as has been the case in previous coronavirus (MERS-CoV and SARS-CoV) epidemics $[44,45]$. Less information exists regarding other broadbased immunosuppressive drugs, such as cyclosporine, methotrexate, mycophenolate, and azathioprine, which are used, alone or in combination with corticosteroids, in a wide range of dermatological diseases.

In summary, the available data on past and present outbreaks of coronavirus suggest that immunosuppressed patients are not at an increased risk for severe disease and complications compared with the general population and that immunosuppressive and immunomodulatory drugs may potentially control the "cytokine storm" associated with a poorer outcome in these patients. Thus, patients with cutaneous immune-mediated diseases (including psoriasis, atopic dermatitis, and hidradenitis suppurativa) can continue their treatment even during the COVID-19 outbreak, preventing disease flares that can contribute to an increasing patient burden, disability, poor quality of life, and healthcare usage.

It is generally recommended to withhold immunosuppressive or biologic treatment in patients with active COVID-19 infection. In patients living in areas with a high incidence of the disease, especially if they develop symptoms consistent with COVID-19 or are close contacts of confirmed cases, individual consideration should be given to the lengthening of administration intervals or temporary treatment discontinuation.

Comorbidities associated with psoriasis, hidradenitis suppurativa, and atopic dermatitis (obesity, cardiovascular disease or risk factors, chronic lung disease, and asthma) and age older than 60 years worsen the prognosis of COVID-19 
infection, and in these cases, treatment interruption and perhaps individual isolation in some circumstances deserve special consideration.

In the future, SARS-Cov-2 vaccination will most likely be added to the immunization recommendations for patients receiving immunosuppressive or biologic treatment for dermatologic indications, including psoriasis, atopic dermatitis, and hidradenitis suppurativa.

Author contribution TT and LP both had the idea for the article, performed the literature search and data analysis, and drafted and critically revised the work.

\section{Compliance with Ethical Standards}

Funding No funding has been received for the preparation of this article.

Conflict of interest Tiago Torres has received consultancy and/or speaker's honoraria from and/or participated in clinical trials sponsored by AbbVie, Amgen, Arena Pharmaceuticals, Boehringer Ingelheim, Bristol Myers Squibb, Celgene, Janssen, Biocad, LEO Pharma, Eli Lilly, MSD, Novartis, Pfizer, Samsung-Bioepis, Sanofi-Genzyme, and Sandoz. Luis Puig has received consultancy and/or speaker's honoraria from and/or participated in clinical trials sponsored by AbbVie, Almirall, Amgen, Baxalta, Biogen, Boehringer Ingelheim, Celgene, Gebro, Janssen, LEO Pharma, Eli Lilly and Company, Merck-Serono, MSD, Mylan, Novartis, Pfizer, Regeneron, Roche, Sandoz, SamsungBioepis, Sanofi, and UCB.

\section{References}

1. European Centre for Disease Prevention and Control. Rapid risk assessment: coronavirus disease 2019 (COVID-19) pandemic: increased transmission in the EU/EEA and the UK-seventh update. 2020. https://www.ecdc.europa.eu/en/publicationsdata/rapid-risk-assessment-coronavirus-disease-2019-covid -19-pandemic. Accessed 1 Apr 2020.

2. World Health Organization. Coronavirus disease (COVID-19) pandemic. https://www.who.int/emergencies/diseases/novel -coronavirus-2019. Accessed 1 Apr 2020.

3. Huang C, Wang Y, Li X, et al. Clinical features of patients infected with 2019 novel coronavirus in Wuhan. China Lancet. 2020;395:497-506. https://doi.org/10.1016/S0140 -6736(20)30183-5.

4. Cascella M, Rajnik M, Cuomo A, et al. Features, evaluation and treatment coronavirus (COVID-19). StatPearls. Treasure Island (FL): StatPearls Publishing. 2020. https://www.ncbi.nlm.nih. gov/books/NBK554776/. Accessed 1 Apr 2020.

5. Fung S-Y, Yuen K-S, Ye Z-W, et al. A tug-of-war between severe acute respiratory syndrome coronavirus 2 and host antiviral defence: lessons from other pathogenic viruses. Emerg Microbes Infect. 2020;9:558-70. https://doi.org/10.1080/22221 751.2020 .1736644 .

6. Prompetchara E, Ketloy C, Palaga T. Immune responses in COVID-19 and potential vaccines: lessons learned from SARS and MERS epidemic. Asian Pac J Allergy Immunol. 2020;38:19. https://doi.org/10.12932/AP-200220-0772.
7. Mehta P, McAuley DF, Brown M, et al. COVID-19: consider cytokine storm syndromes and immunosuppression. Lancet. 2020;395:1033-4. https://doi.org/10.1016/S0140 $-6736(20) 30628-0$.

8. Pedersen SF, Ho Y-C. SARS-CoV-2: a storm is raging. J Clin Invest. 2020. https://doi.org/10.1172/JCI137647.

9. Chen G, Wu D, Guo W, et al. Clinical and immunologic features in severe and moderate coronavirus disease 2019. J Clin Invest. 2020. https://doi.org/10.1172/JCI137244.

10. Qin $\mathrm{C}, \mathrm{Zhou} \mathrm{L}, \mathrm{Hu} \mathrm{Z}$, et al. Dysregulation of immune response in patients with COVID-19 in Wuhan. China. Clin Infect Dis. 2020. https://doi.org/10.1093/cid/ciaa248.

11. Recalcati S. Cutaneous manifestations in COVID-19: a first perspective. J Eur Acad Dermatol Venereol. 2020. https://doi. org/10.1111/jdv.16387.

12. Li H, Zhou Y, Zhang M, et al. Updated approaches against SARS-CoV-2. Antimicrob Agents Chemother. 2020. https:// doi.org/10.1128/AAC.00483-20.

13. Yao X, Ye F, Zhang M, et al. In vitro antiviral activity and projection of optimized dosing design of hydroxychloroquine for the treatment of severe acute respiratory syndrome coronavirus 2 (SARS-CoV-2). Clin Infect Dis. 2020. https://doi.org/10.1093/ $\mathrm{cid} / \mathrm{ciaa} 237$.

14. Zhou D, Dai S-M, Tong Q. COVID-19: a recommendation to examine the effect of hydroxychloroquine in preventing infection and progression. J Antimicrob Chemother. 2020. https:// doi.org/10.1093/jac/dkaa114.

15. Kim AHJ, Sparks JA, Liew JW, et al. A rush to judgment? Rapid reporting and dissemination of results and its consequences regarding the use of hydroxychloroquine for COVID-19. Ann Intern Med. 2020. https://doi.org/10.7326/M20-1223.

16. Zhang W, Zhao Y, Zhang F, et al. The use of anti-inflammatory drugs in the treatment of people with severe coronavirus disease 2019 (COVID-19): the experience of clinical immunologists from China. Clin Immunol. 2020;25(214):108393. https://doi. org/10.1016/j.clim.2020.108393.

17. Sarzi-Puttini P, Ceribelli A, Marotto D, et al. Systemic rheumatic diseases: from biological agents to small molecules. Autoimmun Rev. 2019;18(6):583-92. https://doi.org/10.1016/j. autrev.2018.12.009.

18. Ferro F, Elefante E, Baldini C, et al. COVID-19: the new challenge for rheumatologists. Clin Exp Rheumatol. 2020;38(2):175-80.

19. American Academy of Dermatology Association. Managing your practice through the COVID-19 outbreak. https://www.aad.org/ member/practice/managing/coronavirus. Accessed 1 Apr 2020.

20. International Psoriasis Council. Statement on the coronavirus (COVID-19) outbreak. https://www.psoriasiscouncil.org/blog/ Statement-on-COVID-19-and-Psoriasis.htm. Accessed 1 Apr 2020.

21. AEDV. Comunicado del Grupo de Psoriasis de la AEDV sobre la pandemia por COVID-19. Academia Española de Dermatología y Venereología. 2020. https://aedv.es/comunicado-del-grupo-depsoriasis-de-la-aedv-sobre-la-pandemia-por-covid-19/. Accessed 1 Apr 2020.

22. EULAR. EULAR guidance for patients COVID-19 outbreak. https ://www.eular.org/eular_guidance_for_patients_covid19_outbreak. cfm. Accessed 1 Apr 2020.

23. IOIBD. IOIBD update on COVID19 for patients with Crohn's disease and ulcerative colitis. https://www.ioibd.org/ioibd-updat e-on-covid19-for-patients-with-crohns-disease-and-ulcerative -colitis/. Accessed 1 Apr 2020.

24. Bashyam AM, Feldman SR. Should patients stop their biologic treatment during the COVID-19 pandemic. J Dermatolog Treat. 2020;19:1-2. https://doi.org/10.1080/09546634.2020.1742438. 
25. Lebwohl M, Rivera-Oyola R, Murrell DF. Should biologics for psoriasis be interrupted in the era of COVID-19? J Am Acad Dermatol. 2020. https://doi.org/10.1016/j.jaad.2020.03.031.

26. Price KN, Frew JW, Hsiao JL, et al. COVID-19 and immunomodulator/immunosuppressant use in dermatology. J Am Acad Dermatol. 2020. https://doi.org/10.1016/j.jaad.2020.03.046.

27. Conforti C, Giuffrida R, Dianzani C, et al. COVID-19 and psoriasis: is it time to limit treatment with immunosuppressants? A call for action. Dermatol Ther. 2020;11:e13298. https://doi. org/10.1111/dth.13298.

28. Shah P, Zampella JG. Use of systemic immunomodulatory therapies during the coronavirus disease 2019 (COVID-19) pandemic. J Am Acad Dermatol. 2020. https://doi.org/10.1016/j. jaad.2020.03.056.

29. Favalli EG, Ingegnoli F, De Lucia O, et al. COVID-19 infection and rheumatoid arthritis: faraway, so close! Autoimmun Rev. 2020;20:102523. https://doi.org/10.1016/j.autrev.2020.102523.

30. Russell CD, Millar JE, Baillie JK. Clinical evidence does not support corticosteroid treatment for 2019-nCoV lung injury. Lancet. 2020;395(10223):473-5. https://doi.org/10.1016/S0140 $-6736(20) 30317-2$.

31. Kaltsas A, Sepkowitz K. Community acquired respiratory and gastrointestinal viral infections: challenges in the immunocompromised host. Curr Opin Infect Dis. 2012;25:423-30. https://doi. org/10.1097/QCO.0b013e328355660b.

32. Hui DS, Azhar EI, Kim Y-J, et al. Middle East respiratory syndrome coronavirus: risk factors and determinants of primary, household, and nosocomial transmission. Lancet Infect Dis. 2018;18:e217-e227227. https://doi.org/10.1016/S1473 -3099(18)30127-0.

33. Zhou F, Yu T, Du R, et al. Clinical course and risk factors for mortality of adult inpatients with COVID-19 in Wuhan, China: a retrospective cohort study. Lancet. 2020;395:1054-62. https:// doi.org/10.1016/S0140-6736(20)30566-3.

34. D'Antiga L. Coronaviruses and immunosuppressed patients: the facts during the third epidemic. Liver Transpl. 2020. https://doi. org/10.1002/lt.25756.

35. Fu Y, Cheng Y, Wu Y. Understanding SARS-CoV-2-mediated inflammatory responses: from mechanisms to potential therapeutic tools. Virol Sin. 2020. https://doi.org/10.1007/s12250-02000207-4.
36. Deng X, Yu X, Pei J. Regulation of interferon production as a potential strategy for COVID-19 treatment. https://www.resea rchgate.net/publication/339642124. Accessed 9 Apr 2020.

37. Ma W-T, Yao X-T, Peng Q, et al. The protective and pathogenic roles of IL-17 in viral infections: friend or foe? Open Biol. 2019;9:190109. https://doi.org/10.1098/rsob.190109.

38. Faure E, Poissy J, Goffard A, et al. Distinct immune response in two MERS-CoV-infected patients: can we go from bench to bedside? PLoS ONE. 2014;9:e88716. https://doi.org/10.1371/journ al.pone. 0088716 .

39. Josset L, Menachery VD, Gralinski LE, et al. Cell host response to infection with novel human coronavirus EMC predicts potential antivirals and important differences with SARS coronavirus. mBio. 2013;4:e00165-e1113. https://doi.org/10.1128/ mBio.00165-13.

40. Xu Z, Shi L, Wang Y, et al. Pathological findings of COVID19 associated with acute respiratory distress syndrome. Lancet Respir Med. 2020;8(4):420-2. https://doi.org/10.1016/S2213 -2600(20)30076-X.

41. Hawill B, Geraci J (2020) Repurposing dupilumab may treat advanced COVID-19 patients with severe acute respiratory syndrome by mitigating cytokine storm. https://doi.org/10.31219/osf. io/hk7ep

42. Richardson P, Griffin I, Tucker C, et al. Baricitinib as potential treatment for 2019-nCoV acute respiratory disease. Lancet. 2020;395:e30-e3131. https://doi.org/10.1016/S0140 -6736(20)30304-4.

43. Sanchez GAM, Reinhardt A, Ramsey S, et al. JAK1/2 inhibition with baricitinib in the treatment of autoinflammatory interferonopathies. J Clin Invest. 2018;128:3041-52. https://doi. org/10.1172/JCI98814.

44. Hui DS. Systemic corticosteroid therapy may delay viral clearance in patients with Middle East respiratory syndrome coronavirus infection. Am J Respir Crit Care Med. 2018;197:700-1. https:// doi.org/10.1164/rccm.201712-2371ED.

45. World Health Organization. Clinical management of severe acute respiratory infection when COVID-19 is suspected. https://www. who.int/publications-detail/clinical-management-of-severe-acute -respiratory-infection-when-novel-coronavirus-(ncov)-infectionis-suspected. Accessed 1 Apr 2020. 